Journal of Geodesy (1997) 71: 541-551

Journal of

Geodesy

(C) Springer-Verlag 1997

\title{
On the sensitivity of the location, size and shape of the GPS ambiguity search space to certain changes in the stochastic model
}

\author{
P. J. G. Teunissen \\ Delft Geodetic Computing Centre (LGR), Faculty of Geodesy, Delft University of Technology, Thijsseweg 11, 2629 JA Delft,
} The Netherlands Fax: +31 15278 3711; e-mail: lgr@geo.tudelft.nl

Received: 11 November 1996 / Accepted: 21 March 1997

\begin{abstract}
In this contribution we analyse in a qualitative sense for the geometry-free model the dependency of the location, the size and the shape of the ambiguity search space on different factors of the stochastic model. For this purpose a rather general stochastic model is used. It includes time-correlation, cross-correlation, satellite elevation dependency and the use of an a priori weighted ionospheric model, having the ionospherefixed model and the ionosphere-float model as special cases. It is shown that the location is invariant for changes in the cofactor matrix of the phase observables. This also holds true for the cofactor matrix of the code observables in the ionosphere-float case. As for timecorrelation and satellite elevation dependency, it is shown that they only affect the size of the search space, but not its shape and orientation. It is also shown that the least-squares ambiguities, their variance matrix and its determinant, for, respectively, the ionosphere-fixed model, the ionosphere-float model and the ionosphereweighted model, are all related through the same scalar weighted mean, the weight of which is governed by the variance ratio of the ionospheric delays and the code observables. A closed-form expression is given for the area of the search space in which all contributing factors are easily recognized. From it one can infer by how much the area gets blown up when the ionospheric spatial decorrelation increases. This multiplication factor is largest when one switches from the ionospherefixed model to the ionosphere-float model, in which case it is approximately equal to the ratio of the standard deviation of phase with that of code. The area gives an indication of the number of grid points inside the search space.
\end{abstract}

Key words. GPS $\cdot$ Ambiguity search space $\cdot$ Stochastic model

\section{Introduction}

Integer ambiguity estimation is a prerequisite for fast, high-precision GPS relative positioning. It is based on the integer least-squares principle

$\min _{a}(\hat{a}-a)^{T} Q_{\hat{a}}^{-1}(\hat{a}-a), a$ integer

where $\hat{a}$ is the vector of real-valued least-squares ambiguities and $Q_{\hat{a}}$ its variance matrix. Due to the integer constraints on the double-differenced (DD) ambiguities and the fact that the variance matrix is non-diagonal, the solution of Eq. (1) must be obtained by means of a search. An efficient method for computing the integer least-squares ambiguities has been introduced in Teunissen (1993) and reviewed in Kleusberg and Teunissen (1996); it is the least-squares ambiguity decorrelation adjustment (LAMBDA). Implementation aspects of the method can be found in de Jonge and Tiberius (1996) and de Jonge et al. (1996). The method makes use of the ambiguity search space, which when formulated in the space of the original DD ambiguities is defined as

$(\hat{a}-a)^{T} Q_{\hat{a}}^{-1}(\hat{a}-a) \leq \chi^{2}$

It is located at $\hat{a}$, its shape and orientation are governed by $Q_{\hat{a}}$ and its size can be controlled by $\chi^{2}$.

In this contribution we will study how the location, size and shape of the ambiguity search space are driven by the stochastic model. For this purpose we will make use of a rather general stochastic model. It includes time-correlation, cross-correlation, satellite elevation dependency and a weighting of the ionospheric delays. Our analysis will be based on the geometry-free model (see e.g. Hatch 1982; Melbourne 1985; Wübbena 1985; Euler and Goad 1991; Dedes and Goad 1994; Euler and Hatch 1994; Teunissen 1996). In order to get a deeper insight as to the way in which the different factors of the stochastic model contribute, our analysis will be of a 
qualitative nature rather than a quantitative one. We will therefore put emphasis on analytical results, with, where possible, the formulation of closed-form solutions.

In Sect. 2 we introduce and discuss the functional and stochastic part of the geometry-free model. We distinguish between three versions, the first being the ionosphere-fixed model, the second the ionosphere-float model and the third the ionosphere-weighted model. The first is applicable to short baselines only, whereas the second is applicable to baselines for which the spatial decorrelation of the ionosphere is at its maximum. In Sect. 3 we solve for the location of the search space and show how it depends on the different components of the stochastic model. We also present a recursive formulation, which remains valid in the presence of time-correlation. The dependency of the size and shape of the search space on the stochastic model is discussed in Sect. 4. Here, we distinguish between time-dependent and time-invariant factors. The area of the search space is discussed in Sect. 5; we present a closed-form expression for it and show in which way it is affected by the stochastic model. A summary of our results is given in Sect. 6.

\section{The geometry-free model}

The geometry-free model is the simplest GPS singlebaseline model one can think of that still allows one to determine the integer ambiguities. In this section we will introduce and discuss both its functional and stochastic model. First we consider the single-epoch case, then the multi-epoch case.

\subsection{Single-epoch case}

For a single epoch $i$, the four observation equations of the geometry-free model read

$$
\begin{aligned}
& \phi_{1}(i)=r(i)+\lambda_{1} a_{1}+e_{\phi_{1}}(i) \\
& \phi_{2}(i)=r(i)+\lambda_{2} a_{2}+e_{\phi_{2}}(i) \\
& p_{1}(i)=r(i)+e_{p_{1}}(i) \\
& p_{2}(i)=r(i)+e_{p_{2}}(i)
\end{aligned}
$$

with $\phi_{1}(i)$ and $\phi_{2}(i)$, the DD phase observables on $L_{1}$ and $L_{2}$ at epoch $i$, expressed in units of range, rather than in cycles; $p_{1}(i)$ and $p_{2}(i)$, the corresponding code observables; $r(i)$, the DD form of the unknown ranges from receivers to satellites; $\lambda_{1}$ and $\lambda_{2}$, the known wavelengths of the $L_{1}$ and $L_{2}$ frequency; $a_{1}$ and $a_{2}$, the two unknown integer carrier-phase ambiguities, and, $e_{\phi_{1}}(i), e_{\phi_{2}}(i), e_{p_{1}}(i)$ and $e_{p_{2}}(i)$ the a priori residuals that contain the measurement noises and remaining unmodelled effects.

This model is referred to as geometry-free because it dispenses with the receiver-satellite geometry. The observation equations are therefore linear from the outset and hence no further linearization is needed. Note that the tropospheric delays have not been modelled explic- itly in the equations. The reason for this is that, when present, they would automatically get lumped with the range parameters $r(i)$. This implies that all unknown parameters in the model, except the range parameters $r(i)$, can be estimated free from tropospheric biases. Also note, since the preceding equations are in DD form, that the data are based on using two receivers, both tracking the same two satellites. The two receivers may be in motion or may be stationary.

In the given formulation, the ionospheric delays have been assumed absent, and, it is hence applicable to short baselines only. In case the ionospheric delays are present though, we can still make use of Eq. (3), provided the left sides of the equations are now interpreted as observables being corrected for the ionospheric delays. Hence, in this case we have

$$
\begin{aligned}
& \phi_{1}(i)=\phi_{1}^{\prime}(i)+\mu_{1} I(i) \\
& \phi_{2}(i)=\phi_{2}^{\prime}(i)+\mu_{2} I(i) \\
& p_{1}(i)=p_{1}^{\prime}(i)-\mu_{1} I(i) \\
& p_{2}(i)=p_{2}^{\prime}(i)-\mu_{2} I(i)
\end{aligned}
$$

in which $\mu_{1} I(i)$ denotes the DD ionospheric delay on $L_{1}$ at epoch $i ; \mu_{1}$ and $\mu_{2}$ are the known wavelength ratios $\mu_{1}=\frac{\lambda_{1}}{\lambda_{2}}$ and $\mu_{2}=\frac{\lambda_{2}}{\lambda_{1}}$; and the DD observables are now given by the primed variates.

In this contribution we model the ionospheric delays as random variables. The sample values of the ionospheric delays can be taken from an externally provided ionospheric model, see e.g. Georgiadou (1994), Wild (1994), Wanninger (1995). In some applications where the baselines are sufficiently short it even suffices to take zero as sample value. The a priori uncertainty in the ionospheric delays will be modelled through its variance being proportional to the ionospheric variance factor $s_{I}^{2}$. The use of an a priori weighted ionosphere has been discussed in e.g. Wild and Beutler (1991), Schaer (1994) and Bock (1996). The value of $s_{I}^{2}$ depends to a large extent on the interstation distance between the two receivers. Since the ionosphere decorrelates as function of the interstation distance, $s_{I}^{2}$ is at its maximum for baselines where the ionosphere is fully decorrelated, and it gets smaller the shorter the baselines become. For sufficiently short baselines it can be taken equal to zero. A proposal on how to describe $s_{I}^{2}$ as function of the interstation distance can be found in Bock (1996).

Modelling the ionospheric delays as random variables allows us to consider three versions of the geometry-free model. The version in which the ionospheric delays are assumed absent or known $\left(s_{I}^{2}=0\right)$, the version in which the ionospheric delays are assumed present but completely unknown $\left(s_{I}^{2}=\infty\right)$, and the version in which the ionospheric delays are assumed present and known with uncertainty $\left(0<s_{I}^{2}<\infty\right)$. The first version will be referred to as the ionosphere-fixed model, the second as the ionosphere-float model and the third as the ionosphere-weighted model.

Taking the ionospheric uncertainty into account, the dispersion of $\phi(i)=\left(\phi_{1}(i), \phi_{2}(i)\right)^{T}$ and $p(i)=\left(p_{1}(i)\right.$, $\left.p_{2}(i)\right)^{T}$ is assumed to be proportional to the time-invariant matrix 
$D\left\{\left[\begin{array}{c}\phi(i) \\ p(i)\end{array}\right]\right\} \propto\left[\begin{array}{cc}C_{\phi}+s_{I}^{2} \mu \mu^{T} & -s_{I}^{2} \mu \mu^{T} \\ -s_{I}^{2} \mu \mu^{T} & C_{p}+s_{I}^{2} \mu \mu^{T}\end{array}\right]$

with the cofactor matrices

$$
\begin{gathered}
C_{\phi}=\left[\begin{array}{cc}
c_{\phi_{1}}^{2} & c_{\phi_{1} \phi_{2}} \\
c_{\phi_{2} \phi_{1}} & c_{\phi_{2}}^{2}
\end{array}\right], \quad C_{p}=\left[\begin{array}{cc}
c_{p_{1}}^{2} & c_{p_{1} p_{2}} \\
c_{p_{2} p_{1}} & c_{p_{2}}^{2}
\end{array}\right], \\
\mu \mu^{T}=\left[\begin{array}{cc}
\mu_{1}^{2} & \mu_{1} \mu_{2} \\
\mu_{2} \mu_{1} & \mu_{2}^{2}
\end{array}\right]=\left[\begin{array}{cc}
\left(\lambda_{1} / \lambda_{2}\right)^{2} & 1 \\
1 & \left(\lambda_{2} / \lambda_{1}\right)^{2}
\end{array}\right]
\end{gathered}
$$

Through the two cofactor matrices $C_{\phi}$ and $C_{p}$, we allow the variances of the observables on the two frequencies to differ. Also, the presence of cross-correlation is permitted. Depending on how the measurement process is implemented in the GPS receivers, the observables may or may not be cross-correlated. In the presence of anti-spoofing (AS) for instance, some receivers use a hybrid technique to provide dual-frequency code measurements (Hofmann-Wellenhof et al. 1994). As a result the code data become cross-correlated. For other receivers, $c_{\phi_{1} \phi_{2}}$ and $c_{p_{1} p_{2}}$ can be taken zero.

\subsection{Multi-epoch case}

The functional model can be written in a compact form if we make use of the following matrix-vector notation: $\phi=\left(\phi(1)^{T}, \ldots, \phi(k)^{T}\right)^{T}, \quad p=\left(p(1)^{T}, \ldots, p(k)^{T}\right)^{T}$, $r=(r(1), \ldots, r(k))^{T}, \quad a=\left(a_{1}, a_{2}\right)^{T}, \quad e_{k}=(1, \ldots, 1)^{T}$, $I_{k}=\operatorname{diag}(1, \ldots, 1)$, and $\Lambda=\operatorname{diag}\left(\lambda_{1}, \lambda_{2}\right)$. Using the mathematical expectation $E\{$.$\} and the Kronecker$ product $\otimes$, the linear system of observation equations of the geometry-free model, for $k$ epochs, then reads

$E\left\{\left[\begin{array}{c}\phi \\ p\end{array}\right]\right\}=\left[\begin{array}{cc}I_{k} \otimes e_{2} & e_{k} \otimes \Lambda \\ I_{k} \otimes e_{2} & 0\end{array}\right]\left[\begin{array}{l}r \\ a\end{array}\right]$

Note that the ambiguities are assumed to be constant in time, whereas the DD ranges $r(i)$ are assumed to change from epoch to epoch.

The Kronecker product of two matrices $M$ and $N$ is defined as

$M \otimes N=\left[\begin{array}{ccc}m_{11} N & \ldots & m_{1 q} N \\ \vdots & & \vdots \\ m_{p 1} N & \ldots & m_{p q} N\end{array}\right]$

with $M=\left[m_{i j}\right], i=1, \ldots p, j=1, \ldots, q$. Some of its properties are (see e.g. Rao 1973)

$$
\begin{aligned}
& (M \otimes N)^{T}=M^{T} \otimes N^{T} \\
& (M \otimes N)^{-1}=M^{-1} \otimes N^{-1} \\
& M_{1} M_{2} \otimes N_{1} N_{2}=\left(M_{1} \otimes N_{1}\right)\left(M_{2} \otimes N_{2}\right)
\end{aligned}
$$

As for the stochastic model, the multi-epoch case allows us to take its variability with time into account. This concerns the proportionality factor of Eq. (5). If the precision of the observables is assumed to be time invariant and time-correlation to be absent, this factor is simply a constant. It becomes a function of time how- ever, denoted as $q^{2}(i)$, in case we allow the precision to change with time. And if we also want to allow for the presence of time-correlation, we need to include a coupling between the various epochs as well. This coupling will be denoted as $q(i, j)$. As a result we get for the dispersion

$$
D\left\{\left[\begin{array}{l}
\phi \\
p
\end{array}\right]\right\}=\left[\begin{array}{cc}
Q \otimes\left(C_{\phi}+s_{I}^{2} \mu \mu^{T}\right) & -Q \otimes\left(s_{I}^{2} \mu \mu^{T}\right) \\
-Q \otimes\left(s_{I}^{2} \mu \mu^{T}\right) & Q \otimes\left(C_{p}+s_{I}^{2} \mu \mu^{T}\right)
\end{array}\right]
$$

with the full matrix

$Q=\left[\begin{array}{ccc}q^{2}(1) & \ldots & q(1, k) \\ \vdots & \ddots & \vdots \\ q(k, 1) & \ldots & q^{2}(k)\end{array}\right]$

in which $q^{2}(i)$ takes care of the precision variability over time and where $\rho(i, j)=q(i, j) / q(i) q(j)$ equals the correlation between the two epochs $i$ and $j$.

\subsection{A choice for $q^{2}(i)$ and $q(i, j)$}

Although many of the results obtained in this contribution hold true for an arbitrary choice of the positive definite matrix $Q$, we also consider what happens when a particular choice for this matrix is made. We therefore discuss one possible choice for the matrix $Q$.

In Euler and Goad (1991), Jin (1995) and Gerdan (1995) it has been shown that the time dependency of the precision of the GPS observables can be linked to the change over time of the satellite elevation angles. Furthermore, the decaying exponential function seems to provide an appropriate description for this linkage. We therefore assume that the standard deviation of an undifferenced GPS observable is proportional to

$$
1+c e^{-\epsilon_{r}^{s}(i) / \epsilon_{o}}
$$

where $c$ is a constant, $\epsilon_{r}^{s}(i)$ is the elevation angle under which satellite $s$ is observed from receiver $r$ at epoch $i$, and where $\epsilon_{o}$ is a reference elevation angle. For our DD observables, this description results in

$q^{2}(i)=\sum_{r=1}^{2} \sum_{s=1}^{2}\left(1+c e^{-\epsilon_{r}^{s}(i) / \epsilon_{o}}\right)^{2}$

Note that when the two receivers are sufficiently close together, one may take the two elevation angles to the same satellite as being the same.

In the absence of the satellite elevation dependency we can set $q^{2}(i)=1$ and interpret the entries of the matrix in Eq. (5) as being DD variances and DD covariances. With $q^{2}(i)=1$, matrix $Q$ becomes a correlation matrix, which will be denoted as $R$. A simple model for time-correlation is one where the noise is assumed to follow a first-order autoregressive process. In that case, we have

$R=\left[\rho^{|i-j|}\right], \quad 0 \leq \rho \leq 1$ 
where $\rho$ denotes the correlation coefficient and where $\rho^{|i-j|}$ equals the $(i, j)$ th entry of matrix $R$. The correlation matrix is a unit matrix in case $\rho=0$, and equals the rank-1 matrix $e_{k} e_{k}^{T}$ in case $\rho=1$. For the correlation coefficient one can take $\rho=e^{-T / t}$, with $T$ the sampling interval and $t$ the correlation length. Note that matrix $Q$ can be factored as $Q=\operatorname{diag}[q(1), \ldots, q(k)]$ $R \operatorname{diag}[q(1), \ldots, q(k)]$, in case time-correlation and satellite elevation dependency are both assumed present.

In our analysis we also need the inverse of $Q$ and thus the inverse of the above correlation matrix. Using an upper triangular factorization, it reads

$R^{-1}=L^{T} D L$

with

$L=\left[\begin{array}{cccc}1 & & & \\ -\rho & 1 & & \\ & \ddots & \ddots & \\ & & -\rho & 1\end{array}\right]$,

$D^{-1}=\operatorname{diag}\left[1,\left(1-\rho^{2}\right), \ldots,\left(1-\rho^{2}\right)\right]$
Note that the redundancy of the model equals $3 k-2$. Hence, there is a redundancy of 1 when $k=1$. This is due to having code data on a second frequency as well. For every additional epoch, the redundancy increases by 3 . This is due to the assumed constancy of the two ambiguities and the fact of having code data on two frequencies.

Application of the least-squares principle to this model results in the normal equations

$$
\begin{gathered}
\left(e_{k}^{T} Q^{-1} e_{k} \otimes \Lambda^{T} N^{-1} \Lambda\right) \hat{a}\left(s_{I}^{2}\right)=\left(e_{k}^{T} Q^{-1} \otimes \Lambda^{T} N^{-1}\right)(\phi-p) \\
-\left(e_{k}^{T} Q^{-1} \otimes \Lambda^{T} N^{-1} B C^{-1} d^{T}\right) p
\end{gathered}
$$

with $N=\left(A-B^{T} C^{-1} B\right)$. For the purpose of our further analysis we have explicitly shown the dependence of the least-squares estimate on $s_{I}^{2}$. Solving the normal equations and writing the solution in scalar form gives

$$
\begin{aligned}
& \hat{a}_{1}\left(s_{I}^{2}\right)=\frac{1}{\lambda_{1}} \sum_{i=1}^{k} w_{k}(i)\left[\phi_{1}(i)-\left[w_{11}\left(s_{I}^{2}\right) p_{1}(i)+w_{12}\left(s_{I}^{2}\right) p_{2}(i)\right]\right] \\
& \hat{a}_{2}\left(s_{I}^{2}\right)=\frac{1}{\lambda_{2}} \sum_{i=1}^{k} w_{k}(i)\left[\phi_{2}(i)-\left[w_{21}\left(s_{I}^{2}\right) p_{1}(i)+w_{22}\left(s_{I}^{2}\right) p_{2}(i)\right]\right]
\end{aligned}
$$

Now that we have established our assumptions concerning the stochastic model, we are in the position to determine the least-squares solution of the ambiguities.

\section{The location of the search space}

The location of the search space is uniquely characterized by the least-squares solution of the ambiguities. Since we are only interested in the ambiguities and not in the DD ranges, we first eliminate $r$ from Eq. (6). Premultiplication of Eq. (6) with the $3 k \times 4 k$ matrix

$$
\left[\begin{array}{cc}
I_{k} \otimes I_{2} & -I_{k} \otimes I_{2} \\
0 & I_{k} \otimes d^{T}
\end{array}\right]
$$

with $d=(-1,1)^{T}$ and thus $d^{T} e_{2}=0$, gives together with Eq. (7) the reduced linear system of observation equations

$$
\begin{aligned}
& E\left\{\left[\begin{array}{c}
\phi-p \\
\left(I_{k} \otimes d^{T}\right) p
\end{array}\right]\right\}=\left[\begin{array}{c}
e_{k} \otimes \Lambda \\
0
\end{array}\right] a, \\
& D\left\{\left[\begin{array}{c}
\phi-p \\
\left(I_{k} \otimes d^{T}\right) p
\end{array}\right]\right\}=\left[\begin{array}{cc}
Q \otimes A & Q \otimes B \\
Q \otimes B^{T} & Q \otimes C
\end{array}\right]
\end{aligned}
$$

with

$$
\left\{\begin{array}{l}
A=\left(C_{\phi}+C_{p}+4 s_{I}^{2} \mu \mu^{T}\right) \\
B=-\left(C_{p}+2 s_{I}^{2} \mu \mu^{T}\right) d \\
C=d^{T}\left(C_{p}+s_{I}^{2} \mu \mu^{T}\right) d
\end{array}\right.
$$

with the $k \times 1$ weight vector

$w_{k}=\left(w_{k}(1), \ldots, w_{k}(k)\right)^{T}=Q^{-1} e_{k}\left(e_{k}^{T} Q^{-1} e_{k}\right)^{-1}$

and the $2 \times 2$ matrix of weights

$$
\begin{aligned}
W\left(s_{I}^{2}\right) & =\left[w_{i j}\left(s_{I}^{2}\right)\right] \\
& =I_{2}-\left(C_{p}+2 s_{I}^{2} \mu \mu^{T}\right) d\left[d^{T}\left(C_{p}+s_{I}^{2} \mu \mu^{T}\right) d\right]^{-1} d^{T}
\end{aligned}
$$

The weight vector $w_{k}$ captures the weighting over the epochs, while matrix $W\left(s_{I}^{2}\right)$ gives the within-epoch relative weight of the code observables.

With the solution in Eq. (13) we have one general expression for the least-squares ambiguities. Apart from the data input of course, the solution only changes when the entries of $W\left(s_{I}^{2}\right)$ and/or $w_{k}(i)$ change. Note that they are both independent of the matrix $C_{\phi}$. The leastsquares ambiguity estimates are therefore completely independent of this matrix. This implies that the solution is independent of a possible covariance between the $L_{1}$ and $L_{2}$ carrier phases, that it is also independent of a change in the epoch-wise variance ratio between the $L_{1}$ and $L_{2}$ phase data and that it is independent of the common level over time of the phase variances. Thus the location of the ambiguity search space will not change when matrix $C_{\phi}$ is changed.

This will not be the case of course, when we consider the data input. That is, the location of the ambiguity search space will change when the input data $\phi_{1}(i)$, $\phi_{2}(i), p_{1}(i)$ or $p_{2}(i)$ change. Biases in the data, for instance, will have their effect on the location of the search 
space. If we substitute Eq. (4) into Eq. (13), we can infer the impact of a mismodelling in the ionospheric delays on the location. The bias in the vector of least-squares ambiguities due to biases $b_{I}(i)$ in the ionospheric delays $I(i)$, then follows as

$b_{\hat{a}}\left(s_{I}^{2}\right)=\Lambda^{-1}\left[I_{2}+W\left(s_{I}^{2}\right)\right] \mu \sum_{i=1}^{k} w_{k}(i) b_{I}(i)$

This potential shift in the location of the search space will only be zero when the weighted time average of the ionospheric biases vanishes or when $s_{I}^{2}=\infty$, that is, when the ionosphere-float solution is considered.

The independence of the ambiguity estimates for changes in $C_{\phi}$ implies an invariance for location. Note however, that although the estimates are invariant for such a change, the least-squares ambiguity estimator itself of course will not have this property of invariance. That is, the size and shape of the ambiguity search space will depend on it. We will return to this in Sect. 4. We will now discuss the role of the weights $w_{k}$ and $W\left(s_{I}^{2}\right)$. We also present a recursive formulation for the leastsquares ambiguities.

\subsection{The weight vector $w_{k}$}

The time-dependent part of the stochastic model is captured in the weight vector $w_{k}$. That is, the dependence on matrix $Q$ manifests itself only through the weights $w_{k}(i)$. These weights are independent, however, of $s_{I}^{2}, C_{\phi}$ and $C_{p}$. Since $e_{k}^{T} w_{k}=1$ according to Eq. (14), the weights sum up to unity

$\sum_{i=1}^{k} w_{k}(i)=1$

Also note that in the solution given by Eq. (13), no assumption has yet been made about matrix $Q$. Hence the solution holds for any structure one might choose for matrix $Q$. We will now consider what happens when particular choices are made for the two functions $q(i, j)$ and $q^{2}(i)$.

In case time-correlation is assumed absent, then $q(i, j)=0$ and matrix $Q$ becomes diagonal. In that case we only need to consider the function $q^{2}(i)$. In case it equals unity, then $Q=I_{k}$ and the weights become

$w_{k}(i)=\frac{1}{k} \quad \forall i$

Hence, in this case the linear combinations of the phase and code data are all weighted identically. For a variable function $q^{2}(i)$, defined e.g. according to Eq. (8), we have $Q=\operatorname{diag}\left(q^{2}(1), \ldots, q^{2}(k)\right)$ and the weights become

$w_{k}(i)=\frac{1 / q^{2}(i)}{\sum_{j=1}^{k} 1 / q^{2}(j)}, \quad \forall i$

This implies that more weight is given to the data when $q^{2}(i)$ is small. In case of satellite elevation dependency, it means that more weight is given to the data when the elevation angles are large.

When time-correlation is assumed present, the matrix $Q$ becomes a non-diagonal (full) matrix. Let us first assume $q^{2}(i)=1$. Matrix $Q$ reduces then to a correlation matrix, which equals $R=\left(L^{T} D L\right)^{-1}$, in case a first-order autoregressive process is assumed. With $w_{k}=R^{-1} e_{k}$ $\left(e_{k}^{T} R^{-1} e_{k}\right)^{-1}$, using Eq. (10), the corresponding weights then follow as

$w_{k}(i)=\frac{1}{2 \rho+k(1-\rho)} \times\left\{\begin{array}{cl}1 & \text { for } i=1, k \\ 1-\rho & \text { for } 1<i<k\end{array}\right.$

When we compare these weights with those of Eq. (18), we note that the presence of time-correlation can be mimicked through the use of a function

$q^{2}(i)=\left\{\begin{array}{cl}1+\rho & \text { for } i=1, k \\ \frac{1+\rho}{1-\rho} & \text { for } 1<i<k\end{array}\right.$

If we assume time-correlation to be present and the function $q^{2}(i)$ to vary over time, we have $Q=\operatorname{diag}(q(1)$, $\ldots, q(k))\left(L^{T} D L\right)^{-1} \operatorname{diag}(q(1), \ldots, q(k))$. In this case the weights become

$$
\begin{aligned}
w_{k}(i) & =\frac{1 / q^{2}(i)}{\frac{1-\rho^{2}}{q^{2}(1)}+\sum_{i=2}^{k}\left[\frac{1}{q(i)}-\frac{\rho}{q(i-1)}\right]^{2}} \\
& \times\left\{\begin{array}{cl}
\left(1-\rho \frac{q(i)}{q(i+1)}\right) & \text { for } i=1 \\
\left(1-\rho\left(\frac{q(i)}{q(i-1)}+\frac{q(i)}{q(i+1)}\right)+\rho^{2}\right) & \text { for } 1<i<k \\
\left(1-\rho \frac{q(i)}{q(i-1)}\right) & \text { for } i=k
\end{array}\right.
\end{aligned}
$$

\subsection{The 'weight' matrix $W\left(s_{I}^{2}\right)$}

We will now consider the within-epoch weights and study their dependence on $s_{I}^{2}$ and $C_{p}$. But first note, since $W\left(s_{L}^{2}\right) e_{2}=e_{2}$, that also the entries of the two rows of $W\left(s_{I}^{2}\right)$ sum up to unity

$\left\{\begin{array}{l}w_{11}\left(s_{I}^{2}\right)+w_{12}\left(s_{I}^{2}\right)=1 \\ w_{21}\left(s_{I}^{2}\right)+w_{22}\left(s_{I}^{2}\right)=1\end{array}\right.$

These entries are not necessarily weights in the statistical sense however, since they can take on negative values as well. In the following we will consider, respectively, the ionosphere-fixed solution, the ionosphere-float solution and the ionosphere-weighted solution.

3.2.1. The ionosphere-fixed solution $\left(s_{I}^{2}=0\right)$. In case the ionospheric delays are assumed absent or known, matrix $W\left(s_{I}^{2}\right)$ of Eq. (15) reduces to the projector

$W(0)=I_{2}-C_{p} d\left(d^{T} C_{p} d\right)^{-1} d^{T}$

It projects onto $e_{2}$ and along $C_{p} d$. Since this projector can be alternatively written as $e_{2}\left(e_{2}^{T} C_{p}^{-1} e_{2}\right)^{-1} e_{2}^{T} C_{p}^{-1}$, it immediately follows that 


$$
\begin{aligned}
& w_{11}(0)=w_{21}(0)=\frac{1}{1+\bar{c}_{p_{1}}^{2} / \bar{c}_{p_{2}}^{2}}, \\
& w_{12}(0)=w_{22}(0)=\frac{1}{1+\bar{c}_{p_{2}}^{2} / \bar{c}_{p_{1}}^{2}}
\end{aligned}
$$

with $\bar{c}_{p_{1}}^{2}=c_{p_{1}}^{2}-c_{p_{1} p_{2}}$ and $\bar{c}_{p_{2}}^{2}=c_{p_{2}}^{2}-c_{p_{1} p_{2}}$. This shows that the weights do depend on the presence of covariance between the $L_{1}$ and $L_{2}$ code data. But it also shows that this covariance can be mimicked by using a downscaled variance in case of positive correlation, or by using an up-scaled variance, in case of negative correlation. The expressions for the weights also show that in the absence of covariance, it is the ratio of the $L_{1}$ and $L_{2}$ code variances that counts, and not their individual values.

3.2.2 The ionosphere-float solution $\left(s_{I}^{2}=\infty\right)$. In case the ionospheric delays are assumed present, but completely unknown, matrix $W\left(s_{I}^{2}\right)$ reduces to

$$
W(\infty)=I_{2}-2 \mu \mu^{T} d\left(d^{T} \mu \mu^{T} d\right)^{-1} d^{T}
$$

This matrix is not a projector and its entries, as opposed to the entries of $W(0)$, are both positive and negative. Note that $W(\infty)$ is completely independent of the matrix $C_{p}$. So, the 'weights' are fixed from the outset. Their values ensure that the ambiguity solution is free from the presence of ionospheric biases.

3.2.3 The ionosphere-weighted solution $\left(0<s_{I}^{2}<\infty\right)$. It follows from Eqs. (15), (21) and (22) that matrix $W\left(s_{I}^{2}\right)$ can be written as the linear combination

$W\left(s_{I}^{2}\right)=\alpha W(0)+(1-\alpha) W(\infty)$

with the weight

$\alpha=\frac{1}{1+s_{I}^{2} \mu^{T} P C_{p}^{-1} \mu}$

and the projector $P=d\left(d^{T} C_{p} d\right)^{-1} d^{T} C_{p}$. If we combine Eq. (23) with the solution Eq. (13), it follows that the ionosphere-weighted ambiguity solution can also be written as a linear combination

$\hat{\mathrm{a}}\left(s_{I}^{2}\right)=\alpha \hat{\mathrm{a}}(0)+(1-\alpha) \quad \hat{\mathrm{a}}(\infty)$

This is a very simple expression indeed. It shows that the ionosphere-weighted ambiguity solution is a weighted mean of the ionosphere-fixed solution and the ionosphere-float solution. Moreover, the weights are scalars, which implies that the preceding weighted mean holds for every function of the $L_{1}$ and $L_{2}$ ambiguities. For instance, it holds for the widelane ambiguity or for any one of the transformed ambiguities that are obtained with the LAMBDA method.

The weight $\alpha$ is driven by $s_{I}^{2} \mu^{T} P C_{p}^{-1} \mu$, which equals $s_{I}^{2}\left(\mu_{2}-\mu_{1}\right)^{2} / 2 c_{p}^{2}$ in case $C_{p}=c_{p}^{2} I_{2}$. This shows that the weight $\alpha$ is driven by the ratio of the a priori ionospheric variance and the variance with which the ionospheric delay can be estimated from the code data using a single epoch. In fact when $C_{p}=c_{p}^{2} I_{2}$, then both solutions $\hat{a}(0)$ and $\hat{a}(\infty)$ are independent of $C_{p}$ and the impact of the code precision is then only felt through the ratio $s_{I}^{2} / c_{p}^{2}$ in the weight $\alpha$. This shows that it is not so much the amount of ionospheric decorrelation that determines the weighting, but more its value in relation to the precision of the code data. In other words, improving the precision of the code data has a similar effect as increasing the length of the baseline, as far as the ionospheric delays are concerned.

From the above expression it also follows immediately how a mismodelling of the ionospheric delays propagates as function of $s_{I}^{2}$. Since $b_{\hat{a}}(\infty)=0$, it follows from Eq. (25) that $b_{\hat{a}}\left(s_{I}^{2}\right)=\alpha b_{\hat{a}}(0)$. This shows that the orientation of the bias vector is independent of $s_{I}^{2}$. Only the length of the bias vector depends on $s_{I}^{2}$.

\subsection{A recursive formulation}

The least-squares solution given by Eq. (13) is given in batch form; we now present a recursive formulation for it, expressed in the predictor-corrector form. First however, we write Eq. (13) in a more compact form. The least-squares ambiguity solution based on data from a single epoch, say epoch $i$, is given as

$$
\begin{aligned}
& a_{1}(i)=\frac{1}{\lambda_{1}}\left[\phi_{1}(i)-\left[w_{11}\left(s_{I}^{2}\right) p_{1}(i)+w_{12}\left(s_{I}^{2}\right) p_{2}(i)\right]\right] \\
& a_{2}(i)=\frac{1}{\lambda_{2}}\left[\phi_{2}(i)-\left[w_{21}\left(s_{I}^{2}\right) p_{1}(i)+w_{22}\left(s_{I}^{2}\right) p_{2}(i)\right]\right]
\end{aligned}
$$

Note that we have omitted the argument $s_{I}^{2}$ and have replaced it instead with the argument of time $i$. With $a(i)=\left(a_{1}(i), a_{2}(i)\right)^{T}$, we can now write Eq. (13) as

$\hat{a}_{k \mid k}=\sum_{i=1}^{k} w_{k}(i) a(i)$

Note that we have used a double subindex for time. As with the usual Kalman-filter notation, this is to distinguish between the predictor (time update) and the corrector (measurement update). It will be clear that Eq. (26) does not admit a recursive formulation in general, unless the weights satisfy certain properties. That is, a recursive formulation is not possible for an arbitrary positive definite matrix $Q$. It is possible, however, when matrix $Q$ is of the form $Q=\operatorname{diag}(q(1), \ldots, q(k))\left(L^{T} D L\right)^{-1} \operatorname{diag}(q(1), \ldots, q(k))$. A recursive formulation is thus possible even in the presence of time-correlation. To show this, first note that we have the following recursion for $w_{i}(i)$,

$$
\left\{\begin{aligned}
n(1) & =\frac{q^{2}(1)}{1-\rho^{2}}, \quad w_{1}(1)=1 \\
m(i) & =1-\rho \frac{q(i)}{q(i-1)} \quad i \geq 2 \\
n(i) & =\left[1 / n(i-1)+m^{2}(i) / q^{2}(i)\right]^{-1} \quad i \geq 2 \\
w_{i}(i) & =n(i) m(i) / q^{2}(i) \quad i \geq 2
\end{aligned}\right.
$$


It can be verified using Eq. (20). Similarly, it follows that

$$
\begin{aligned}
& w_{k}(i) \\
& =\left\{\begin{array}{cc}
\left(1-w_{k}(k) m(k)\right) w_{k-1}(i) & \text { for } 1 \leq i<k-1 \\
\left(1-w_{k}(k) m(k)\right) w_{k-1}(i)-w_{k}(k)(1-m(k)) & \text { for } i=k-1
\end{array}\right.
\end{aligned}
$$

This together with Eq. (26) shows that

$$
\begin{aligned}
\hat{a}_{k \mid k}= & \hat{a}_{k-1 \mid k-1}+w_{k}(k) \\
& \times\left[a(k)-\left[m(k) \hat{a}_{k-1 \mid k-1}+(1-m(k)) a(k-1)\right]\right]
\end{aligned}
$$

In this expression, we recognize the term inside the inner square brackets as the one-step-ahead least-squares predictor of the ambiguities. It will be denoted as $\hat{a}_{k \mid k-1}$. As a result, the predictor-corrector recursion follows for $k \geq 2$ as

$$
\begin{aligned}
\hat{a}_{k \mid k-1} & =a(k-1)-m(k)\left[a(k-1)-\hat{a}_{k-1 \mid k-1}\right] \\
\hat{a}_{k \mid k} & =\hat{a}_{k-1 \mid k-1}+w_{k}(k)\left[a(k)-\hat{a}_{k \mid k-1}\right]
\end{aligned}
$$

For $k=2$, we have $a(1)=\hat{a}_{1 \mid 1}=\hat{a}_{2 \mid 1}$. Note that $a(k-1)-\hat{a}_{k-1 \mid k-1}$ is the least-squares ambiguity residual at epoch $k-1$, where as $a(k)-\hat{a}_{k \mid k-1}$ is the predicted ambiguity residual at epoch $k$. Also note that the prediction step is absent, $\hat{a}_{k \mid k-1}=\hat{a}_{k-1 \mid k-1}$, when $m(k)=1$, which happens when time-correlation is absent $(\rho=0)$.

\section{The size and shape of the search space}

In the previous section we considered the location of the ambiguity search space. In this section we consider its size and shape. The size and shape of the ambiguity search space is completely captured by the variancecovariance matrix of the least-squares ambiguities. If we invert the normal matrix of Eq. (12), or apply the error propagation law to Eq. (13), the ambiguity variance matrix follows as

its shape or orientation. This is significant, since it implies that also the decorrelating ambiguity transformation of the LAMBDA method is not affected by it. That is, one will get the same transformed ambiguities, whether matrix $Q$ equals the identity matrix or not. If we assume time-correlation to follow a first-order autoregressive process, the scale factor equals

$\frac{1}{e_{k}^{T} Q^{-1} e_{k}}=\left[\frac{1}{q^{2}(1)}+\frac{1}{1-\rho^{2}} \sum_{i=2}^{k}\left[\frac{1}{q(i)}-\frac{\rho}{q(i-1)}\right]^{2}\right]^{-1}$

Hence, for $q(i)=1$ we get

$\frac{1}{k} \leq \frac{1}{e_{k}^{T} Q^{-1} e_{k}}=\frac{1}{1+(k-1) \frac{1-\rho}{1+\rho}} \leq 1$

where the bounds are reached for, respectively, $\rho=0$ and $\rho=1$. This shows that when $\rho>0$ the size of the search space will decrease less rapidly as time progresses than when time-correlation is absent. In fact, the size will not change at all when $\rho=1$.

\subsection{Time-invariant shape}

Since all time-dependent variables are captured in the scale factor $1 / e_{k}^{T} Q^{-1} e_{k}$, it follows from Eq. (29) that the shape and orientation of the search space are themselves time invariant. Hence, once the decorrelating ambiguity transformation of the LAMBDA method has been computed, it remains applicable irrespective of the observation time span or sampling rate used. For its computation, only $s_{I}^{2}$ and the two matrices $C_{\phi}$ and $C_{p}$ need to be known.

4.2.1 Ionosphere-fixed solution. The ambiguity variance matrix for the ionosphere-fixed solution follows from substituting $s_{I}^{2}=0$ into Eq. (29) as

$Q_{\hat{a}}(0)=\frac{1}{e_{k}^{T} Q^{-1} e_{k}} \Lambda^{-1}\left[C_{\phi}+C_{p}\left(I_{2}-P\right)\right] \Lambda^{-T}$

As opposed to the ambiguity estimates which were discussed in the previous section, this result shows that the stochastic model does have a complete and direct impact on the ambiguity variance matrices and thus also on the geometry of the ambiguity search spaces. There is, however, a distinct difference as to their impact on the size and shape of the search space.

\subsection{Time-dependent size}

It follows from Eq. (29) that the influence of the matrix $Q$ is only felt through the scale factor $1 / e_{k}^{T} Q^{-1} e_{k}$. Hence, matrix $Q$ only affects the size of the search space and not where matrix $\Lambda=\operatorname{diag}\left(\lambda_{1}, \lambda_{2}\right)$ contains the wavelengths on $L_{1}$ and $L_{2}$. Note, since $C_{p}\left(I_{2}-P\right)=e_{2}\left(e_{2}^{T} C_{p}^{-1} e_{2}\right)^{-1} e_{2}^{T}$, that the variance matrix equals the sum of a full-rank matrix and a rank-1 matrix. The entries of the full-rank matrix are very small compared to the entries of the rank-1 matrix. From this structure and the fact that the phase data are much more precise than the code data follows immediately that the vector $\Lambda d$ is a good approximation of the eigenvector that belongs to the smallest eigenvalue. The vector orthogonal to $\Lambda d$, which is $\left(\lambda_{2},-\lambda_{1}\right)^{T}$, is therefore a good approximation of the eigenvector belonging to the largest eigenvalue. This shows that the major principal axis of the search space is located in the first and third quadrant, having an 
orientation of less than 45 degrees. In fact, to a good approximation, the orientation of the ionosphere-fixed search space can be shown to equal 38 degrees.

The shape of the search space can be measured by its elongation $e$. It equals the length ratio of the major and minor principal axes of the search space. It is therefore equal to the square root of the condition number of the ambiguity variance matrix. The elongation of the ionosphere-fixed search space, $e(0)$, follows to a first-order approximation from Eq. (31) as

$e(0) \approx \frac{1}{2}\left(\mu_{1}+\mu_{2}\right) \frac{c_{p}}{c_{\phi}}$

which equals 103 when $c_{p} / c_{\phi}=10^{2}$. This shows that the search space is very elongated indeed.

4.2.2 Ionosphere-float solution. The ambiguity variance matrix for the ionosphere-float solution follows from substituting $s_{I}^{2}=\infty$ into Eq. (29) as

$$
\begin{aligned}
Q_{\hat{a}}(\infty) & =\frac{1}{e_{k}^{T} Q^{-1} e_{k}} \Lambda^{-1} \\
& \times\left[C_{\phi}+C_{p}+\frac{2\left(\mu \mu^{T}\left(I_{2}-P\right)+\left(I_{2}-P\right)^{T} \mu \mu^{T}\right)}{\mu^{T} P C_{p}^{-1} \mu}\right] \Lambda^{-T}
\end{aligned}
$$

The orientation of the corresponding search space follows to a good approximation as 44.8 degrees. Note that in contrast with the ionosphere-fixed case, the ambiguity variance matrix now remains of full rank, even when $C_{\phi}=0$. Hence, the search space will not collapse into a straight line when the phase data are assumed to be perfectly known. In fact, the elongation of the search space is to a first-order approximation independent of the phase-code variance ratio,

$e(\infty) \approx\left(\mu_{1}+\mu_{2}\right)\left(1+\frac{8}{\left(\mu_{2}-\mu_{1}\right)^{2}}\right) \approx 67$

The search space is therefore still elongated, but less so than in the ionosphere-fixed case.

4.2.3 Ionosphere-weighted solution. If we combine Eqs. (31) and (33) with Eq. (29), it follows that apart from the least-squares solution itself, also the ambiguity variance matrix of the ionosphere-weighted solution can be written as a weighted mean

$$
Q_{\hat{a}}\left(s_{I}^{2}\right)=\alpha Q_{\hat{a}}(0)+(1-\alpha) Q_{\hat{a}}(\infty)
$$

This implies that the shape and orientation of the ionosphere-weighted search space interpolates between the values given for, respectively, the ionosphere-fixed search space and the ionosphere-float search space. And since the weights are scalars, the same weighted mean is taken of the variance matrices when one works with ambiguities other than the DD ambiguities. For instance, when instead of the DD ambiguity vector $a$, the ambiguity vector $z=Z^{T} a$ is used, with matrix $Z$ being integer and area-preserving, we would have $Q_{\hat{z}}\left(s_{I}^{2}\right)=\alpha Q_{\hat{z}}(0)+(1-\alpha) Q_{\hat{z}}(\infty)$.

\section{Area of search space}

The area of the ambiguity search space, denoted by $S$, is given for the ionosphere-weighted case as

$S\left(s_{I}^{2}\right)=\pi \chi^{2} \sqrt{\left|Q_{\hat{a}}\left(s_{I}^{2}\right)\right|}$

where $\left|Q_{\hat{a}}\left(s_{I}^{2}\right)\right|$ is the determinant of the ambiguity variance matrix. The area gives an indication of the number of grid points that are located inside the search space (Teunissen 1993). Numerical examples showing how well this approximation works are given in Teunissen et al. (1996). Note that the area, and thus the determinant as well, is invariant for any areapreserving transformation. Since all admissible ambiguity transformations are members from this class (Teunissen 1995), it follows that the determinant (or its square root) of the ambiguity variance matrix gives a truly intrinsic description of the precision of the ambiguities.

\subsection{A closed-form expression}

We will now derive a closed-form expression for the area of the search space. In the previous section we treated the time-dependent and time-invariant effects separately. In case of the area however, all of the elements of the stochastic model, such as $Q, s_{I}^{2}, C_{\phi}$ and $C_{p}$, will contribute. We will therefore factor the solution for the area in such a way that the various contributing factors are clearly recognized.

If we invert the normal matrix of Eq. (12) and take the determinant we get

$\left|Q_{\hat{a}}\left(s_{I}^{2}\right)\right|=\frac{1}{\left(e_{k}^{T} Q^{-1} e_{k}\right)^{2}} \frac{1}{\lambda_{1}^{2} \lambda_{2}^{2}}\left|A-B^{T} C^{-1} B\right|$

We will now solve for the determinant $\left|A-B^{T} C^{-1} B\right|$ in a few consecutive steps. First we note that the matrix

$\left[\begin{array}{cc}A & B \\ B^{T} & C\end{array}\right]=X+s_{I}^{2} y y^{T}$

with

$X=\left[\begin{array}{cc}C_{\phi}+C_{p} & -C_{p} d \\ -d^{T} C_{p} & d^{T} C_{p} d\end{array}\right], \quad y=\left[\begin{array}{c}2 \mu \\ -\mu^{T} d\end{array}\right]$

is the sum of a full-rank matrix $X$ and a rank-1 matrix $y y^{T}$. This implies that the determinant of the matrix on the right side of Eq. (38), equals $|X|\left(1+s_{I}^{2} y^{T} X^{-1} y\right)$. The determinant of the matrix on the left side follows after orthogonalization as $\left|A-B^{T} C^{-1} B \| C\right|$. Hence, we have

$\left|A-B^{T} C^{-1} B\right|=|X|\left(1+s_{I}^{2} y^{T} X^{-1} y\right)|C|^{-1}$ 
The last two terms in this expression follow rather straightforwardly as
This result allows us now to infer in a rather straightforward manner the impact of the weighting of the

$$
\begin{aligned}
|C| & =d^{T}\left(C_{p}+s_{I}^{2} \mu \mu^{T}\right) d \\
1+s_{I}^{2} y^{T} X^{-1} y & =\left(1+s_{I}^{2} \mu^{T} P C_{p}^{-1} \mu\right)\left(1+4 s_{I}^{2} \frac{\mu^{T}\left[\left(I_{2}+P\right)^{T} C_{\phi}\left(I_{2}+P\right)+\left(I_{2}-P\right)^{T} C_{p}\left(I_{2}-P\right)\right]^{-1} \mu}{1+s_{I}^{2} \mu^{T} P C_{p}^{-1} \mu}\right)
\end{aligned}
$$

For the first term on the right side, we have

$$
\begin{aligned}
|X| & =\left|C_{\phi}+C_{p}\left(I_{2}-P\right) \| d^{T} C_{p} d\right| \\
& =\left|C_{\phi}+e_{2}\left(e_{2}^{T} C_{p}^{-1} e_{2}\right)^{-1} e_{2}^{T} \| d^{T} C_{p} d\right| \\
& =\left|C_{\phi}\right|\left(1+\frac{e_{2}^{T} C_{\phi}^{-1} e_{2}}{e_{2}^{T} C_{p}^{-1} e_{2}}\right)\left|d^{T} C_{p} d\right|
\end{aligned}
$$

The first equality follows from taking the determinant of $X$ after its first two rows are orthogonalized with respect to the third. The second equality follows from substituting the projector $P=I_{2}-C_{p}^{-1} e_{2}\left(e_{2}^{T} C_{p}^{-1} e_{2}\right)^{-1} e_{2}^{T}$ and the third equality follows from taking the determinant of a rank-1 updated matrix. If we now collect our results and substitute them into Eq. (37), the sought-for expression for the determinant follows as ionosphere on the area of the search space. In order to do so, we first consider the area of the search space for the two cases $s_{I}^{2}=0$ and $s_{I}^{2}=\infty$.

5.2.1 Ionosphere-fixed solution. When $C_{\phi}=c_{\phi}^{2} I_{2}$ and $C_{p}=c_{p}^{2} I_{2}$, the determinant of the ionosphere-fixed solution follows from Eq. (40) as

$$
\left|Q_{\hat{a}}(0)\right|=\left(e_{k}^{T} Q^{-1} e_{k}\right)^{-2} \frac{c_{\phi}^{4}}{\lambda_{1}^{2} \lambda_{2}^{2}}\left(1+\frac{c_{p}^{2}}{c_{\phi}^{2}}\right)
$$

This shows, since $c_{p} \gg c_{\phi}$, that we have to a good approximation

$$
\left|Q_{\hat{a}}\left(s_{I}^{2}\right)\right|=\underbrace{\frac{1}{\left(e_{k}^{T} Q^{-1} e_{k}\right)^{2}}}_{1} \underbrace{\frac{C_{\phi} \mid}{\lambda_{1}^{2} \lambda_{2}^{2}}}_{2} \underbrace{\left(1+\frac{e_{2}^{T} C_{\phi}^{-1} e_{2}}{e_{2}^{T} C_{p}^{-1} e_{2}}\right)}_{3} \underbrace{\left(1+4 s_{I}^{2} \frac{\mu^{T}\left[\left(I_{2}+P\right)^{T} C_{\phi}\left(I_{2}+P\right)+\left(I_{2}-P\right)^{T} C_{p}\left(I_{2}-P\right)\right]^{-1} \mu}{1+s_{I}^{2} \mu^{T} P C_{p}^{-1} \mu}\right)}_{4}
$$

The first factor depends on time, whereas the remaining three factors are time invariant. The first two factors equal the determinant if the code data are perfectly known. This determinant would be extremely small due to the high precision of the phase data. In that case ambiguity validation would almost become trivial. The third factor enters when one considers the ionospherefixed solution for $C_{p} \neq 0$. This factor is very much larger than 1 , since $C_{p} \gg C_{\phi}$. It shows that in the geometryfree model, ambiguity validation heavily depends on the precision of the code data. In fact, the third factor would become infinite in the absence of code data, thus showing that in the geometry-free model, code data are needed per se. Finally, the fourth factor enters when the ionospheric delays are taken into account. This factor is also greater than 1 and increases when $s_{I}^{2}$ gets larger, thus showing that the area of the search space increases for increasing baseline lengths.

\subsection{The determinant as a weighted mean}

When we substitute $s_{I}^{2}=0$ or $s_{I}^{2}=\infty$ into Eq. (40), it follows upon inspection that also the determinant of the ionosphere-weighted solution can be written as a weighted mean

$$
Q_{\hat{a}}\left(s_{I}^{2}\right)|=\alpha| Q_{\hat{a}}(0)|+(1-\alpha)| Q_{\hat{a}}(\infty) \mid
$$

$\left|Q_{\hat{a}}(0)\right| \approx\left(e_{k}^{T} Q^{-1} e_{k}\right)^{-2} \frac{c_{\phi}^{2} c_{p}^{2}}{\lambda_{1}^{2} \lambda_{2}^{2}}$

Hence, apart from the time-dependent part, it is the product of the standard deviations of phase and code that governs the area of the ionosphere-fixed search space.

5.2.2 Ionosphere-float solution. When $C_{\phi}=c_{\phi}^{2} I_{2}$ and $C_{p}=c_{p}^{2} I_{2}$, the determinant of the ionosphere-float solution follows from Eq. (40) as

$$
\begin{aligned}
\left|Q_{\hat{a}}(\infty)\right|= & \left(e_{k}^{T} Q^{-1} e_{k}\right)^{-2} \frac{c_{\phi}^{4}}{\lambda_{1}^{2} \lambda_{2}^{2}}\left(1+\frac{c_{p}^{2}}{c_{\phi}^{2}}\right) \\
& \times\left(1+\frac{c_{p}^{2}}{c_{\phi}^{2}}\left(1+4\left[1+\frac{c_{p}^{2}}{c_{\phi}^{2}}\right]^{-1} \frac{\left(\mu_{2}+\mu_{1}\right)^{2}}{\left(\mu_{2}-\mu_{1}\right)^{2}}\right)\right)
\end{aligned}
$$

This shows, since $c_{p} \gg c_{\phi}$, that we have to a good approximation

$\left|Q_{\hat{a}}(\infty)\right| \approx\left(e_{k}^{T} Q^{-1} e_{k}\right)^{-2} \frac{c_{p}^{2} c_{p}^{2}}{\lambda_{1}^{2} \lambda_{2}^{2}}$

Hence, apart from the time-dependent part, it is now not the product of the standard deviations of phase and 
code that governs the area of the search space, but the standard deviation of code with itself.

If we compare Eq. (43) with Eq. (45), it follows that

$$
\left|Q_{\hat{a}}(\infty)\right| /\left|Q_{\hat{a}}(0)\right| \approx \frac{c_{p}^{2}}{c_{\phi}^{2}}
$$

This shows that when one goes from the ionospherefixed solution to the ionosphere-float solution, the area of the search space gets blown up by a factor that equals the ratio of the standard deviation of phase with that of code. In practice this factor is about 100 . Hence, the ionosphere-float ambiguity search space will admit about 100 times more grid points than its ionospherefixed counterpart. This explains why it is so much more difficult to get the ambiguities validated in the ionosphere-float case than in the ionosphere-fixed case.

5.2.3 Ionosphere-weighted solution. Now that we know the order of magnitudes of the two determinants $\left|Q_{\hat{a}}(0)\right|$ and $\left|Q_{\hat{a}}(\infty)\right|$, we can make use of the weighted mean Eq. (41) to infer the sensitivity of the area with respect to the spatial decorrelation of the ionosphere. We will take the area of the ionosphere-fixed search space as reference. We therefore write

$S\left(s_{I}^{2}\right)=\lambda(\alpha) S(0)$ with $\lambda(\alpha)=\sqrt{\alpha+(1-\alpha) S(\infty)^{2} / S(0)^{2}}$

According to Eq. (46), $S(\infty)^{2} / S(0)^{2} \approx c_{p}^{2} / c_{\phi}^{2}$, which in practice is about $10^{4}$, and according to Eq. (24) we have for the weight $\alpha=\left[1+\left(s_{I}^{2} / c_{p}^{2}\right)\left(\mu_{2}-\mu_{1}\right)^{2} / 2\right]^{-1}$ when $C_{\phi}=c_{\phi}^{2} I_{2}$ and $C_{p}=c_{p}^{2} I_{2}$. Table 1 shows, for different values of the weight $\alpha$, by how much the area of the ionosphere-fixed search space gets blown up. It shows that $\alpha$ should not be too far from 1 in order not to have too large a blown-up area. In terms of the spatial decorrelation, it shows that already when $s_{I}$ equals a few times $c_{p}$, the area gets close to its maximum value. The area blows up by half of its maximum value when $s_{I} / c_{p}=1.6$.

\section{Summary}

In this contribution we considered the geometry-free model and studied the dependence of the location, size and shape of the ambiguity search space on the stochastic model. The stochastic model included timecorrelation, cross-correlation and satellite elevation dependency.

Table 1. Sensitivity of search space area for spatial decorrelation

\begin{tabular}{lll}
\hline$\alpha$ & $s_{I} / c_{p}$ & $\lambda(\alpha)=S\left(s_{I}^{2}\right) / S(0)$ \\
\hline 0.125 & 7.4 & 94 \\
0.25 & 4.8 & 87 \\
0.5 & 2.8 & 71 \\
0.75 & 1.6 & 50 \\
0.8 & 1.4 & 45 \\
0.9 & 0.9 & 32 \\
0.99 & 0.3 & 10 \\
\hline
\end{tabular}

Solutions were derived for the least-squares ambiguities, their variance matrix and its determinant. It was shown that their respective solutions for the ionospherefixed model, the ionosphere-float model and the ionosphere-weighted model are all related through the same weighted mean

$\hat{a}\left(s_{I}^{2}\right)=\alpha \hat{a}(0)+(1-\alpha) \hat{a}(\infty)$

$Q_{\hat{a}}\left(s_{I}^{2}\right)=\alpha Q_{\hat{a}}(0)+(1-\alpha) Q_{\hat{a}}(\infty)$

$\left|Q_{\hat{a}}\left(s_{I}^{2}\right)\right|=\alpha\left|Q_{\hat{a}}(0)\right|+(1-\alpha)\left|Q_{\hat{a}}(\infty)\right|$

The first equation governs the location of the search space, the second governs its shape and orientation, and the third governs the area of the search space.

The dependence of the location on the stochastic model could be separated into a time-dependent part and a time-invariant part. The time-dependent part manifests itself through the weights $w_{k}(i)$. They are the same for both $\hat{a}_{1}$ and $\hat{a}_{2}$, and more generally, for each pair of admissible ambiguities. We also presented a recursive formulation for the least-squares ambiguities. It applies also in the presence of time-correlated autoregressive noise.

For the time-invariant dependence of the location on the stochastic model, we discriminated between the ionosphere-fixed case, the ionosphere-float case and the ionosphere-weighted case. The only commonality is that all three types of solution are completely invariant for changes in the cofactor matrix $C_{\phi}$ of the phase observables. The ionosphere-float location was shown to be invariant for changes in $C_{p}$ as well. For the ionospherefixed location this only holds true when $C_{p}=c_{p}^{2} I_{2}$. In general however, it does depend on $C_{p}$. In this case, it was shown that the covariance between the two code observables can be mimicked by adjusting the level of their variances. The dependence on $s_{I}^{2}$ of the ionosphereweighted location manifests itself through the weight $\alpha$. Here however, it is the ratio $s_{I} / c_{p}$ that counts, and not so much their individual values.

As to the size and shape of the ambiguity search space, it was shown that time-correlation and satellite elevation dependency manifest themselves only through a scale factor of the ambiguity variance matrix. This holds true for all three versions of the geometry-free model. Hence, only the size of the search space is affected, but not its shape and orientation. It implies for instance, that when time-correlation is erroneously assumed absent the computed size of the search space will decrease more rapidly than it actually should. It also implies that the performance of the LAMBDA method, as to its ability to obtain decorrelated ambiguities, is not affected by the presence of time-correlation and/or satellite elevation dependency. But a mismodelling of timecorrelation and satellite elevation dependency does affect, of course, the ambiguity validation process.

It was shown that the impact of the stochastic model on the shape and orientation of the search space was felt only through $s_{I}^{2}$ and the two matrices $C_{\phi}$ and $C_{p}$. Since both the shape and orientation of the search spaces are time invariant, the shape and orientation of the trans- 
formed search spaces as obtained with the LAMBDA method are time invariant as well. Due to the given weighted mean, the elongation $e\left(s_{I}^{2}\right)$ and orientation $\theta\left(s_{I}^{2}\right)$ of the ionosphere-weighted search space interpolate between the ones of the ionosphere-fixed case and the ionosphere-float case. Thus: $67 \leq e\left(s_{I}^{2}\right) \leq 103$ and $38^{\circ} \leq \theta\left(s_{I}^{2}\right) \leq 44.8^{\circ}$.

The area of the search space gives an indication of the number of grid points inside the search space. It is based on the determinant of the ambiguity variance matrix, which itself, due to its invariance against admissible ambiguity transformations, gives an intrinsic description of the ambiguity precision. A closed-form expression was derived for this determinant. This expression was factored in such a way that all contributing factors could be easily recognized. The area of the ionosphere-fixed search space was shown to be almost proportional to $c_{\phi} c_{p}$. Its ionosphere-float counterpart, however, was shown to be almost proportional to $c_{p} c_{p}$. The factor by which the area gets blown up when the ionospheric delays are not assumed to be absent or known, is therefore maximally $c_{p} / c_{\phi}$. This explains why it is so much more difficult to get the ambiguities validated for long baselines. Through the weighted mean of the determinants, it was also shown by how much this factor gets reduced when use is made of an a priori weighted ionosphere.

Acknowledgements. This work was finalized during the author's stay as guest professor at the Department of Geodesy and Geomatics Engineering of the University of New Brunswick (Fredericton, Canada), with Professor A. Kleusberg as his host.

\section{References}

Bock Y (1996) Medium-distance GPS measurements. In: Kleusberg A, Teunissen PJG (eds) GPS for Geodesy. Lecture Notes in Earth Sciences Vol. 60. Springer, Berlin Heidelberg New York, Chapter 9

Dedes G, Goad C (1994) Real-time cm-level GPS positioning of cutting blade and earth movement equipment. In: Proc ION-94 National Meeting, San Diego, California. pp 587-594

de Jonge PJ, Tiberius CCJM (1996) The LAMBDA method for integer ambiguity estimation: implementation aspects. LGR Series 12, Delft Geodetic Computing Centre

de Jonge PJ, Tiberius CCJM, Teunissen PJG (1996) Computational aspects of the LAMBDA method for GPS ambiguity resolution. In: Proc ION GPS-96, Kansas City, Missouri, Sept. $17-20,10 \mathrm{p}$
Euler H, Goad C (1991) On optimal filtering of GPS dual-frequency observations without using orbit information. Bull Geod 65: 130-143

Euler H, Hatch R (1994) Comparison of several AROF kinematic techniques. In: Proc ION-94. pp 363-370

Georgiadou Y (1994) Modelling the ionosphere for an active control network of GPS stations. LGR Series 7, Delft Geodetic Computing Centre

Gerdan GP (1995) A comparison of four methods of weighting double-difference pseudo-range measurements. Trans Tasman Surveyor, 1: 60-66

Hatch R (1982) The synergism of GPS code and carrier phase measurements. In: Proc 3rd Int Geod Symp Satellite Positioning, Las Vegas, New Mexico, Vol. 2, pp 1213-1231

Hofmann-Wellenhof B, Lichtenegger H, Collins J (1994) GPS Theory and Practice (3rd edn) Springer; Wien

Jin XX (1995) A recursive procedure for computation and quality control of GPS differential corrections. LGR Series 8, Delft Geodetic Computing Centre

Kleusberg A, Teunissen PJG (eds) (1996) GPS for Geodesy. Lecture Notes in Earth Sciences Vol 60. Springer, Berlin Heidelberg New York

Melbourne WG (1985) The case for ranging in GPS-based geodetic systems. In: 1st Int Symp Precise Positioning with the Global Positioning System, Rockville, MD, USA. pp 373-386

Rao CR (1973) Linear Statistical Inference and its Applications. Wiley, New York

Schaer S (1994) Stochastische Ionosphärenmodellierung beim 'Rapid Static Positioning' mit GPS. Astronomisches Institut, Universität Bern

Teunissen PJG (1993) Least-squares estimation of the integer GPS ambiguities. LGR Series 6, Delft Geodetic Computing Centre

Teunissen PJG (1995) The invertible GPS ambiguity transformations. Manuscr Geod 20: 489-497

Teunissen PJG (1996) An analytical study of ambiguity decorrelation using dual-frequency code and carrier phase. J Geod 70: 515-528

Teunissen PJG, de Jonge PJ, Tiberius CCJM (1996) The volume of the GPS ambiguity search space and its relevance for integer ambiguity resolution. In: Proc ION GPS-96, Kansas City, Missouri, Sept. 17-20. pp 889-898

Wanninger L (1995) Improved ambiguity resolution by regional differential modelling of the ionosphere. In: Proc ION GPS-95, September 12-15, 1995. pp 889-898

Wild U (1994) Ionosphere and geodetic satellite systems: permanent GPS tracking data for modelling and monitoring. Geod Geophys Arb Schweiz, Band 48

Wild U, Beutler G (1991) Deterministische und stochastische Ionosphärenmodelle. Z Vermessung, Photogr Kulturtech 6: 298-302

Wübbena G (1985) Software developments for geodetic positioning with GPS using TI 4100 code and carrier measurements. In: 1st Int Symp precise Positioning with the Global Positioning System, Rockville, MD, USA, pp 403-412 